\title{
HER2 expression in renal cell carcinoma is rare and negatively correlated with that in normal renal tissue
}

\author{
HUILI WANG ${ }^{1 *}$, CHENGYI LIU ${ }^{2 *}$, JUN HAN $^{2}$, LIN ZHEN $^{3}$, TAO ZHANG ${ }^{1}$, \\ XIAOLI HE ${ }^{1}$, ENCI XU ${ }^{4}$ and MENGQIANG $\mathrm{LI}^{2}$ \\ ${ }^{1}$ Department of Immunology, Fujian Medical University; ${ }^{2}$ Department of Urology, \\ The Union Hospital of Fujian Medical University; ${ }^{3}$ Department of Pathology; \\ ${ }^{4}$ Institute of Urology, Fujian Medical University, Fuzhou, P.R. China
}

Received February 1, 2012; Accepted May 11, 2012

DOI: $10.3892 / \mathrm{ol} .2012 .727$

\begin{abstract}
The aim of this study was to evaluate the status of HER2 protein expression in patients with renal cell carcinoma (RCC) and to determine its prognostic significance. A total of 42 paraffin-embedded tumor tissues and 42 additional corresponding adjacent normal tissues from RCC patients were randomly collected and studied using immunohistochemistry (IHC). Protein samples of 6 fresh specimens from tumor and adjacent normal tissues obtained during surgery were extracted and tested using western blotting to confirm the IHC results. Of the 42 tumor tissues and adjacent normal tissues tested, IHC showed that 7 tumors (16.67\%) and 33 adjacent normal tissues $(78.57 \%)$ expressed the HER2 protein. In addition, results of the western blotting revealed weak HER2 reactivity in primary tumor cells in two of 6 specimens obtained during surgery. All 6 normal tissues showed positive expression, which was in accordance with the outcome of IHC. In conclusion, HER2 is frequently expressed in normal renal tissues and rarely expressed in RCC tissues. Furthermore, the HER2 status of normal tissue is negatively correlated with that of the RCC tissues $(\mathrm{r}=-0.410, \mathrm{P}=0.007)$ and the TNM stage $(r=-0.246, P=0.027)$, suggesting that HER2 is involved in RCC oncogenesis.
\end{abstract}

Correspondence to: Dr Enci Xu, Institute of Urology, Fujian Medical University, XinQuan Road No. 29, Fuzhou, Fujian 350001, P.R. China

E-mail: encixu@yahoo.cn

Dr Mengqiang Li, Department of Urology, The Union Hospital of Fujian Medical University, XinQuan Road No. 29, Fuzhou, Fujian 350001, P.R. China

E-mail: limengqiang1976@163.com

*Contributed equally

Key words: HER2, renal cell carcinoma, TNM stage

\section{Introduction}

Renal cell carcinoma ( RCC) is one of the most common types of malignant tumor of the human urinary system. To date, the benefit of conventional therapies for RCC, including surgical, radiological and chemotherapeutic approaches, is limited. Treatment with IFN and IL-2 remains the main immunotherapy method for RCC after surgery treatment. The efficacy rate is $10-20 \%$ when IFN is used alone to treat metastatic RCC (1). Therefore, a more effective potential therapy needs to be found. New targeted therapy for RCC may open up a new avenue for cancer treatment and targeted therapy depends on the evaluation of target gene status.

HER2, or ErbB-2, is a member of the epidermal growth factor receptor (EGFR) family with intrinsic protein tyrosine kinase activity and its increased activity is the assumed mechanism underlying cell transformation (2). HER2 combines with the other EGFRs to form heterogeneous dimers and is involved in signal transduction, cell proliferation, development, differentiation, migration and tumor formation (3). Previous studies have reported that HER2-positive status was an independent predictor of poor prognosis in multivariate analysis (4). Herceptin, which is targeted against the HER2 cell-surface receptor, has been successfully used for the treatment of breast cancer. At present, there are conflicting reports concerning HER2 expression in RCC due to different laboratory conditions, case groups or the ethnicity of patients. In the present study, we evaluated the HER2 status of 42 RCC tumor and normal tissue specimens using immunohistochemistry (IHC) and 6 specimens using western blotting. Unlike the overexpression observed in breast cancer, IHC showed that HER2 is commonly expressed in normal renal, rather than RCC tissues. Since it has been found that HER2 is expressed by the normal adult kidney, the presence of this oncoprotein in the normal kidney may affect the possibility of using HER2-targeted therapy for the treatment of RCCs overexpressing HER2. The present study represents the rationale of the analysis.

\section{Materials and methods}

Study population and tissue specimens. A total of 84 paraffin-embedded specimens, including 42 tumor tissues and 
42 corresponding adjacent normal tissues, obtained during a two-year period (between January 2009 and December 2010) and provided by The Union Hospital of Fujian Medical University (Fuzhou, China), were analyzed to identify HER2 immunohistochemically stained sections in renal carcinoma cases. Of these cases, 37 patients had clear cell renal carcinoma, 3 had papillary renal carcinoma and 2 had carcinoma of the collecting ducts. Adjacent normal tissues were also identified from the RCC nephrectomy specimens. A total of 6 patients with RCC, who were histologically diagnosed following surgery and treated in the Department of Urology, The Union Hospital of Fujian Medical University, China, were enrolled in a protein extraction and western blotting study to verify the HER2 IHC expression. The study was approved by the Institutional Ethics committee of the Union Hospital of Fujian Medical University, and written informed consent was obtained from the participants.

IHC analysis. Immunohistochemical staining was performed using HER2/ErbB2 (29D8) rabbit mAb (dilution 1:100, Cell Signaling Technology, Beverly, MA, USA). Briefly, the slides were rehydrated and antigen retrieval was achieved by microwave for $15 \mathrm{~min}$ in citrate buffer. The slides were incubated in $3 \%$ hydrogen peroxide to quench endogenous horseradish peroxidase (HRP) for $30 \mathrm{~min}$, followed by incubation with normal goat serum in PBS for $60 \mathrm{~min}$ at room temperature. The slides were then incubated with the primary antibody at $4^{\circ} \mathrm{C}$ overnight. Subsequently, the slides were incubated with biotin-labeled anti-rabbit IgG and preformed avidin-biotin peroxidase complex. The slides were then counterstained with hematoxylin, dehydrated and mounted.

For analysis of HER2 staining, the tumor images were collected at a magnification of $\mathrm{x} 400$ and the proportion of positively stained nuclei was determined for a minimum of 5 fields of view. The integrated optical density (IOD) was then measured using Image-Pro plus 5.0 software. The expression of HER 2 of the 42 specimens was classified by two pathologists in our institute using the semiquantitative scoring (SQS) recommended in UK guidelines in separate settings. In this setting, these guidelines define a positive HER2 status as: IHC score of $3+$, uniform, intense membranous staining in $>30 \%$ of the tumor; $2+$, weak to moderate complete membranous staining that is non-uniform or weak in intensity in at least $10 \%$ of the cells; 1+, weak and incomplete membranous staining in $<10 \%$ of the tumor cells; a negative HER2 status is defined as an IHC score of 0 , no staining. Hematoxylin and eosin staining of the slides of tumors was performed to show the area of the tumor or adjacent normal tissues and then scanned using a microscope (Nikon TE2000-U, Tokyo, Japan).

Western blotting. Fresh specimens, including tumor tissues and corresponding adjacent normal tissues, were obtained during surgery. A total of 6 RCC tissues, including 1 papillary renal carcinoma, 5 clear cell renal carcinomas and their normal adjacent tissues, were used. Cell membrane proteins were extracted using a Mem-PER Eukaryotic Membrane Protein Extraction kit (Thermo Fisher Scientific Inc., Logan, UT, USA). For the western blotting analysis, crude membrane pellets were solubilized in electrophoresis sample buffer and boiled for $10 \mathrm{~min}$, then separated on an $8 \%$ gel. Following electrophoresis, the proteins were loaded onto a polyvinylidene fluoride (PVDF) microporous membrane (Millipore, Billerica, MA, USA). After blocking of non-specific binding with 5\% bovine serum albumin (BSA) for $2 \mathrm{~h}$ at room temperature, the proteins were subsequently identified using a primary antibody specific to HER2 (dilution 1:1,000) in PBST under gentle agitation at $4^{\circ} \mathrm{C}$ overnight. Western blots were developed with the secondary antibody anti-rabbit IgG (dilution 1:5,000; Bioss, Beijing, China) and enhanced chemiluminescence (ECL; Amersham Pharmacia, Freiburg, Germany) detection system. $\beta$-actin was used as a loading control.

Statistical analysis. The Mann-Whitney U test was used to analyze the statistical contrast between HER2 expression in $\mathrm{RCC}$ and normal tissues. The correlation coefficients ( $\mathrm{r}$ and P-values) between the HER2 status of normal tissue and the TNM stage were obtained using the Spearman test. $\mathrm{P}<0.05$ was considered to indicate a statistically significant result. Statistical analyses were performed using SPSS 11.5 software (SPSS, Inc., Chicago, IL, USA).

\section{Results}

The clinical and demographic details of the 42 patients are shown in Table I, based on TNM classification. HER2 expression was assessed generally by IHC. Our aim was to determine the frequency of HER 2 expression in RCC and corresponding normal tissue and the correlation between HER2 expression and tumor grading. A distinctive HER2 expression in renal normal tissue was confirmed at the protein level by IHC. The sections of tumor and adjacent normal tissues are shown in Fig. 1. Of a total of 42 tumor and adjacent normal tissues, HER2 expression was observed in 7 of the 42 tumors (16.67\%) using IHC. For the majority of the tumors, HER2 expression was negative. However, 33 adjacent normal tissues $(78.57 \%)$ expressed the HER2 protein (Table II), which was confined to the renal tubule and renal collecting duct as shown in Fig. 1. This is an unfavorable outcome contrary to our intent.

To confirm this result, we investigated the HER2 expression in 6 fresh RCC and adjacent normal tissues by western blotting. Of the 6 tumor and adjacent normal tissues, only 2 cases of tumor tissue expressed HER 2 weakly. However, the adjacent normal tissues exhibited positive expression (Fig. 2). This outcome is in accordance with the results previously described in the paraffin-embedded tissues. Therefore, HER2 is mainly expressed in normal kidney tissue specimens, rather than in RCC tissues. To examine the change in HER2 expression during RCC pathogenesis, we analyzed the correlation between normal renal tissue and tumor tissue. The HER2 status of normal tissue was negatively correlated with that of the RCC tissues ( $\mathrm{r}=-0.410, \mathrm{P}=0.007$; Table II). Furthermore, the HER2 status of normal tissue was negatively correlated with the TNM stage $(\mathrm{r}=-0.246, \mathrm{P}=0.027)$ following statistical analysis according to the difference in stage (Table III).

\section{Discussion}

The incidence rate of RCC, including clear cell renal cell carcinoma (70-80\%), papillary renal cell carcinoma (10-15\%), chromophobe renal cell carcinoma (5\%) and carcinoma of the 
Table I. Clinical data of 42 patients with RCC.

\begin{tabular}{|c|c|c|c|c|c|c|c|}
\hline \multirow[b]{2}{*}{ Case } & \multirow[b]{2}{*}{ Gender } & \multirow[b]{2}{*}{ Age (years) } & \multirow[b]{2}{*}{ Clinical stage } & \multirow[b]{2}{*}{ Tumor size $(\mathrm{cm})$} & \multirow[b]{2}{*}{ TNM stage } & \multicolumn{2}{|c|}{ HER2 expression } \\
\hline & & & & & & RCC tissues & Normal tissues \\
\hline 1 & $\mathrm{~F}$ & 63 & 1 & 3 & T1N0M0 & 0 & $3+$ \\
\hline 2 & M & 43 & 1 & 3.5 & T1N0M0 & 0 & $3+$ \\
\hline 3 & M & 61 & 1 & 2.9 & T1N0M0 & 0 & $3+$ \\
\hline 4 & $\mathrm{~F}$ & 57 & 1 & 4.3 & T1N0M0 & 0 & $3+$ \\
\hline 5 & M & 38 & 1 & 6.0 & T1N0M0 & $2+$ & 0 \\
\hline 6 & M & 63 & 1 & 4.3 & T1N0M0 & 0 & $1+$ \\
\hline 7 & M & 59 & 2 & 8.5 & T2N0M0 & $3+$ & $1+$ \\
\hline 8 & $\mathrm{~F}$ & 25 & 1 & 4.3 & T1N0M0 & 0 & $3+$ \\
\hline 9 & M & 17 & 4 & 10 & T4N1M0 & 0 & 0 \\
\hline 10 & M & 55 & 1 & 3.6 & T1N0M0 & $2+$ & 0 \\
\hline 11 & $\mathrm{~F}$ & 60 & 1 & 3.5 & T1N0M0 & 0 & $3+$ \\
\hline 12 & $\mathrm{~F}$ & 56 & 2 & 10 & T2N0M0 & $2+$ & $1+$ \\
\hline 13 & $\mathrm{~F}$ & 58 & 2 & 13.1 & T2N0M0 & 0 & $3+$ \\
\hline 14 & M & 61 & 1 & 0.9 & T1N0M0 & 0 & 0 \\
\hline 15 & $\mathrm{M}$ & 60 & 1 & 4.1 & T1N0M0 & $3+$ & 0 \\
\hline 16 & $\mathrm{M}$ & 46 & 1 & 4.3 & T1N0M0 & 0 & $2+$ \\
\hline 17 & M & 62 & 3 & 5.9 & T3bN1M0 & 0 & $3+$ \\
\hline 18 & $\mathrm{M}$ & 31 & 1 & 2.2 & T1N0M0 & 0 & $1+$ \\
\hline 19 & M & 69 & 3 & 2.4 & T3N0M0 & 0 & $1+$ \\
\hline 20 & $\mathrm{~F}$ & 60 & 3 & 8.6 & T2N1M0 & 0 & $3+$ \\
\hline 21 & $\mathrm{~F}$ & 58 & 2 & 9.9 & T2N0M0 & 0 & 0 \\
\hline 22 & M & 65 & 1 & 6.5 & T1N0M0 & 0 & $3+$ \\
\hline 23 & $\mathrm{M}$ & 67 & 3 & 1.7 & T3N0M0 & 0 & $2+$ \\
\hline 24 & $\mathrm{M}$ & 40 & 1 & 2.2 & T1N0M0 & 0 & $3+$ \\
\hline 25 & M & 70 & 3 & 2.5 & T2N1M0 & 0 & $3+$ \\
\hline 26 & M & 61 & 3 & 1.2 & T3N0M0 & 0 & 0 \\
\hline 27 & $\mathrm{M}$ & 48 & 2 & 13.6 & T2N0M0 & 0 & $2+$ \\
\hline 28 & $\mathrm{M}$ & 47 & 3 & 4.5 & T3N1M0 & 0 & 0 \\
\hline 29 & $\mathrm{M}$ & 44 & 1 & 6.0 & T1N0M0 & $1+$ & $3+$ \\
\hline 30 & $\mathrm{M}$ & 47 & 1 & 1.7 & T1N0M0 & 0 & $3+$ \\
\hline 31 & $\mathrm{M}$ & 43 & 1 & 4.8 & T1N0M0 & 0 & $3+$ \\
\hline 32 & M & 50 & 1 & 3.0 & T1N0M0 & 0 & $3+$ \\
\hline 33 & $\mathrm{M}$ & 53 & 1 & 4.2 & T1N0M0 & 0 & $2+$ \\
\hline 34 & $\mathrm{M}$ & 41 & 1 & 4.5 & T1N0M0 & 0 & $2+$ \\
\hline 35 & $\mathrm{M}$ & 69 & 1 & 1.5 & T1N0M0 & 0 & $3+$ \\
\hline 36 & $\mathrm{~F}$ & 62 & 1 & 5.9 & T1N0M0 & 0 & $3+$ \\
\hline 37 & M & 45 & 1 & 6.1 & T1N0M0 & 0 & $2+$ \\
\hline 38 & $\mathrm{M}$ & 51 & 4 & 14.0 & $\mathrm{~T} 2 \mathrm{~N} 2 \mathrm{M} 0$ & 0 & 0 \\
\hline 39 & $\mathrm{~F}$ & 62 & 1 & 4.5 & T1N0M0 & 0 & $3+$ \\
\hline 40 & $\mathrm{~F}$ & 45 & 1 & 5.2 & T1N0M0 & $2+$ & $3+$ \\
\hline 41 & $\mathrm{M}$ & 48 & 2 & 3.8 & T2N0M0 & 0 & $2+$ \\
\hline 42 & $\mathrm{~F}$ & 52 & 1 & 5.7 & T1N0M0 & 0 & $2+$ \\
\hline
\end{tabular}

RCC, renal cell carcinoma; M, male; F, female.

collecting ducts (1\%), comprises $90-95 \%$ of all neoplasms of the kidney. As a malignant tumor, RCC has a poor prognosis, due in large part to the fact that $40 \%$ of patients develop distant metastases following removal of the primary tumor and that available chemotherapeutic agents are ineffective against RCC (5). Thus, there is a need for the development of new treatment strategies. In recent years, molecular targeted therapy has become a new treatment modality for RCC. 
Table II. The correlation between HER2 expression in RCC and normal tissue.

\begin{tabular}{|c|c|c|c|c|c|c|c|}
\hline & \multicolumn{4}{|c|}{ HER2 } & \multirow[b]{2}{*}{ Total } & \multirow[b]{2}{*}{$\mathrm{r}$} & \multirow[b]{2}{*}{ P-value } \\
\hline & - & + & ++ & +++ & & & \\
\hline $\mathrm{RCC}$ & 35 & 1 & 4 & 2 & 42 & -0.410 & 0.007 \\
\hline Normal & 9 & 5 & 8 & 20 & 42 & & \\
\hline
\end{tabular}

RCC, renal cell carcinoma.
Table III. HER2 status of normal tissue is negatively correlated with the TNM stage.

\begin{tabular}{|c|c|c|c|c|c|c|}
\hline \multirow[b]{2}{*}{ TNM } & \multicolumn{4}{|c|}{ Normal tissue } & \multirow[b]{2}{*}{$\mathrm{r}$} & \multirow[b]{2}{*}{ P-value } \\
\hline & - & + & ++ & +++ & & \\
\hline $\mathrm{T} 1$ & 4 & 2 & 5 & 16 & -0.246 & 0.027 \\
\hline $\mathrm{T} 2$ & 2 & 2 & 2 & 3 & & \\
\hline $\mathrm{T} 3$ & 2 & 1 & 1 & 1 & & \\
\hline $\mathrm{T} 4$ & 1 & 0 & 0 & 0 & & \\
\hline
\end{tabular}

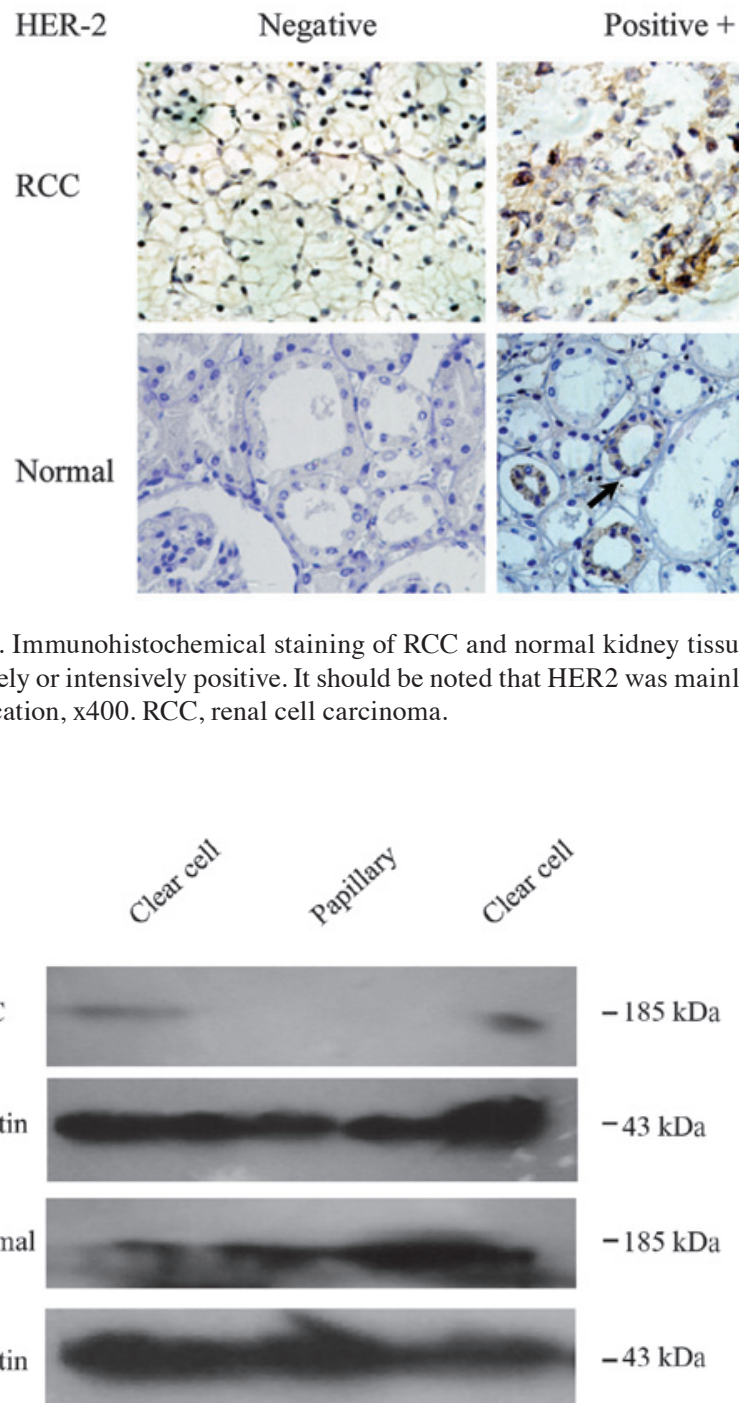

Figure 2. The verification of the HER2 expression using western blotting. HER 2 expression in adjacent normal tissues was found to be higher than that in RCC tissues. RCC, renal cell carcinoma.

Herceptin, which is targeted against the HER2 cell-surface receptor, has been reported to increase the cure rate of breast cancer by $20 \%(6,7)$. HER 2 has been identified as a potential therapeutic target. However, molecular targeted therapy for RCC depends on the evaluation of the target gene status.

HER2 amplification and overexpression play significant roles in signal transduction, cell proliferation, development, differentiation, migration and tumor formation. Findings of available reports indicate a discrepancy with regard to HER2 expression in RCC. It has been reported that the HER2 positive rate in RCC is $40 \%$ (8). However, other authors have found that the positive rate of HER2 expression was $11 \%$ in RCC tissues and was expressed at heterogeneous levels independently of tumor grading and staging (9). Another study reported that the HER2 gene was neither overexpressed nor amplified in cases of Wilms tumor (10). This discrepancy may partly be explained by differences in staining technique and patient cohorts $(11,12)$. Results of the present study emphasize the low rate of expression and complete concordance between IHC and western blotting analyses in RCC. Therefore, although a high positive rate was observed in breast cancer, gastric carcinoma and bladder cancer $(13,14)$, HER 2 expression in RCC may be rare, as in endometrial cancer (15). It is not appropriate to employ antibody- or CTL-based immunotherapy, such as antibody-coated tumor cell vaccine, in cases of RCC (16).

Notably, in our study there was a negative correlation between the HER 2 expression in normal tissue and that of RCC $(\mathrm{P}=0.007, \mathrm{r}=-0.410)$. Coincidently, the location where HER2 was expressed in normal tissues was the same site from which RCC later originated. Taken together, this finding indicates that a decrease in the expression of this gene occurred during RCC oncogenesis. Furthermore, for the patients in whom RCC had already occurred, the HER2 status of the 
normal tissue was negatively correlated with the TNM stage to a certain extent $(\mathrm{P}=0.027, \mathrm{r}=-0.246)$, which also confirms that HER2 is involved in RCC oncogenesis. This observation suggests that HER2 expression was reduced during the process of RCC oncogenesis and also played a significant role in RCC development later, indicating a new mechanism of HER2 in the progression of RCC. In conclusion, HER2 is not an important target for the treatment of RCC. In fact, since tumors which overexpressed HER2 showed negative adjacent normal tissues, HER2 in this specific patient subgroup could serve as a candidate treating target.

\section{Acknowledgements}

This study was partly supported by grants from the Fujian Natural Sciences Foundation (2009J05069, 2009J05067) and Fujian Education Department Foundation (JA09113). We gratefully acknowledge Professor Yinghong Yang (Department of Pathology, The Union Hospital of Fujian Medical University) for the generous and critical advice.

\section{References}

1. Fossa SD: Interferon in metastatic renal cell carcinoma. Semin Oncol 27: 187-193, 2000.

2. Klapper LN, Glathe S, Vaisman N, et al: The ErbB-2/HER2 oncoprotein of human carcinomas may function solely as a shared coreceptor for multiple stroma-derived growth factors. Proc Natl Acad Sci USA 96: 4995-5000, 1999.

3. Reese DM and Slamon DJ: HER-2/neu signal transduction in human breast and ovarian cancer. Stem Cells 15: 1-8, 1997.

4. Revillion F, Bonneterre J and Peyrat JP: ERBB2 oncogene in human breast cancer and its clinical significance. Eur J Cancer 34: 791-808, 1998.
5. Motzer RJ, Bander NH and Nanus DM: Renal-cell carcinoma. N Engl J Med 335: 865-875, 1996.

6. Disis ML, Wallace DR, Gooley TA, et al: Concurrent trastuzumab and HER2/neu-specific vaccination in patients with metastatic breast cancer. J Clin Oncol 27: 4685-4692, 2009.

7. Tokugawa T, Kobayashi A, Matsuyama T, Imai S and Koyama H: A patient with axillary node metastasis from breast cancer who responded to trastuzumab/capecitabine combination therapy. Gan To Kagaku Ryoho 36: 467-469, 2009 (In Japanese).

8. Zhang XH, Takenaka I, Sato C and Sakamoto H: p53 and HER-2 alterations in renal cell carcinoma. Urology 50: 636-642, 1997.

9. Ragab SM, Samaka RM and Shams TM: HER2/neu expression: a predictor for differentiation and survival in children with Wilms tumor. Pathol Oncol Res 16: 61-67, 2010.

10. Vasei M, Modjtahedi H, Ale-Booyeh O, et al: Amplification and expression of EGFR and ERBB2 in Wilms tumor. Cancer Genet Cytogenet 194: 88-95, 2009.

11. Latif Z, Watters AD, Bartlett JM, Underwood MA and Aitchison M: Gene amplification and overexpression of HER2 in renal cell carcinoma. BJU Int 89: 5-9, 2002.

12. Seliger B, Rongcun Y, Atkins D, et al: HER-2/neu is expressed in human renal cell carcinoma at heterogeneous levels independently of tumor grading and staging and can be recognized by HLA-A2.1-restricted cytotoxic T lymphocytes. Int J Cancer 87: 349-359, 2000.

13. Laé M, Couturier J, Oudard S, Radvanyi F, Beuzeboc P and Vieillefond A: Assessing HER2 gene amplification as a potential target for therapy in invasive urothelial bladder cancer with a standardized methodology: results in 1005 patients. Ann Oncol 21: 815-819, 2010.

14. Shiroiwa T, Fukuda T and Shimozuma K: Cost-effectiveness analysis of trastuzumab to treat HER2-positive advanced gastric cancer based on the randomised ToGA trial. Br J Cancer 105: 1273-1278, 2011.

15. Srijaipracharoen S, Tangjitgamol S, Tanvanich S, et al: Expression of ER, PR, and Her-2/neu in endometrial cancer: a clinicopathological study. Asian Pac J Cancer Prev 11: 215-220, 2010.

16. Wang H, Wang D, Li M, et al: Enhanced anti-tumor immunity generated by Rituximab-coated tumor cell vaccine. Cancer Lett 268: 129-136, 2008. 